\title{
ARTINIAN AND NOETHERIAN HYPERCENTRAL GROUPS
}

\section{HERMANN SIMON}

1. Recently, Kemhadze [1] has proved that a finite group $G$ is nilpotent if and only if each nonabelian subgroup $S$ of $G$ has a noncyclic commutator factorgroup. In this note we will generalize Kemhadze's theorem in two ways: the first two theorems will be concerned with artinian groups which contain Kemhadze's theorem as a special case; our third theorem will characterize noetherian nilpotent groups in a similar way.

I want to take this opportunity to express my appreciation to Professor Reinhold Baer for his interest in the completion of this paper.

\section{Notations.}

artinian $=$ minimum condition for subgroups.

noetherian $=$ maximum condition for subgroups.

factor of $G=$ any epimorphic image of any subgroup of $G$.

$Z(G)=$ center of $G$.

$Z_{0}=1 \leqq Z_{1}=Z(G) \leqq \cdots \leqq Z_{\alpha} \leqq \cdots$ are the terms of the upper central series of $G$ (possibly continued transfinitely).

hypercenter $=$ last term of the upper central series.

hypercentral i.e. $G$ itself is a term of its upper central series.

nilpotent i.e. $G=Z_{n}, n$ a natural number.

class of $G=$ smallest integer $n$ (provided that it does exist) such that $G=Z_{n}$.

$x \circ y=x^{-1} y^{-1} x y$.

$A \circ B=$ subgroup of $G$ generated by $a \circ b$ where $a \in A$ and $b \in B$. ${ }^{0} G=G \geqq{ }^{1} G=G \circ G \geqq{ }^{2} G=G \circ{ }^{1} G \geqq \cdots \geqq{ }^{\alpha} G \geqq \cdots$ are the terms of the lower central series (possibly continued transfinitely).

$p=$ natural prime number.

3. In the following lemma $\leftleftarrows$ will be a group-theoretical property such that if $A$ and $B$ are two normal (E-subgroups of the group $G$ then $A B$ is also a (normal) (E-subgroup of $G$.

LEMMA. If $M$ is a group which is not an E-group but all whose proper normal subgroups are 5 -groups, then $M / M^{\prime}$ is either a finite cyclic p-group or a Prüfer group of type $p^{\infty}$.

Proof. Since the product of any two proper normal subgroups of

Received by the editors January 21, 1966. 
$M$ is a proper subgroup of $M$, the result follows by Newman-Wiegold [1, p. 244].

In Theorems 1 and 2 below we will apply this lemma in case $\mathbb{E}=$ hypercentral (according to P. Hall [1, Lemma 1, p. 334] "hypercentral" meets the requirement for $(\xi)$ and $(\xi=$ nilpotent.

Theorem 1. The following properties of the artinian group $G$ are equivalent:

(I) $G$ is hypercentral.

(II) If $S$ is a nonabelian finitely generated subgroup of $G$ and if $S / S^{\prime}$ is primary, then $S / S^{\prime}$ is noncyclic.

Proof. (I) $\Rightarrow(\mathrm{II})$. According to Baer [3, Satz 4.1, p. 21] $G$ is locally finite-and-nilpotent; hence (II) is a consequence of (I).

(II) $\Rightarrow(\mathrm{I})$. Deny. Since $G$ is artinian, there exists a subgroup $M$ of $G$ which is not hypercentral but whose proper subgroups are hypercentral: in particular $M$ is not abelian. If $M$ were not finitely generated, each finitely generated subgroup $F$ of $M$ would be hypercentral. Since $G$ and a fortiori $F$ are artinian, $F$ would be finite and nilpotent, so that the artinian group $M$ is locally finite-and-nilpotent and therefore by Baer [3, Satz 4.1, p. 21] $M$ is hypercentral, a contradiction. Therefore $M$ is finitely generated. According to the preceding lemma, $M / M^{\prime}$ is a cyclic $p$-group. Since $M$ is not abelian, this is a contradiction. Q.E.D.

After deleting "finitely generated" from (II) in Theorem 1 one obtains

THEOREM 2. The following properties of the artinian group $G$ are equivalent:

(I) $G$ is nilpotent

(II) If $S$ is a nonabelian subgroup of $G$ and if $S / S^{\prime}$ is primary, then $S / S^{\prime}$ is noncyclic.

Proof. (I) $\Rightarrow(\mathrm{II})$. Clear.

$(\mathrm{II}) \Rightarrow(\mathrm{I})$. Deny. Since $G$ is artinian, there exists a subgroup $M$ of $G$ which is not nilpotent, but whose proper subgroups are nilpotent. By (II) $M / M^{\prime}$ is not cyclic so that according to the preceding lemma $M / M^{\prime}$ is a Prüfer group of type $p^{\infty}$. By Theorem 1 the artinian group $M$ is hypercentral; by Baer [3, Satz 4.1, p. 21] there exists an abelian normal subgroup $A \triangleleft M$ with finite $M / A$. Since $M / A M^{\prime}$ is a finite factorgroup of the Prüfer group $M / M^{\prime}, M=A M^{\prime}$. Since $M^{\prime} \triangleleft M$, $M^{\prime}$ is nilpotent and hence $A M^{\prime}=M$ is nilpotent, a contradiction. Q.E.D. 
Theorem 3. $G$ is noetherian and nilpotent if and only if (a) $G$ is finitely generated, (b) each nonabelian factor of $G$ has a noncyclic commutator factorgroup and (c) there exists an integer $n \geqq 0$ such that the class of a finite nilpotent factorgroup of $G$ does not exceed $n$.

Proof. The necessity of (a), (b) and (c) is readily seen.

Now assume the validity of (a), (b) and (c) and deny that $G$ is noetherian and nilpotent. Then by Baer [1, Lemma 4, p. 410] there exists an epimorphic image $H$ of $G$ which is not of finite class, but whose proper epimorphic images are of finite class.

(1) $H$ does not contain abelian normal subgroups $\neq 1$.

Assume there exists an abelian normal subgroup $A \neq 1$ of $H$. Since $H / A$ is finitely generated and nilpotent, by Baer [2, Satz 1, p. 310] $H$ is nilpotent, a contradiction.

(2) ${ }^{\omega} H=\bigcap_{i=0}^{\infty}{ }^{i} H=1$.

Deny. Apply (b) to see that $\left({ }^{\omega} H\right)^{\prime} \neq{ }^{\omega} H \neq 1$ and apply (1) to show $\left({ }^{\omega} H\right)^{\prime} \neq 1$. Hence $H /\left({ }^{\omega} H\right)^{\prime}$ is of finite class, proving the existence of a positive integer $c$ with the property ${ }^{\omega} H \leqq{ }^{c} H \leqq\left({ }^{\omega} H\right)^{\prime}<{ }^{\omega} H$, the desired contradiction.

(3) Since ${ }^{\omega} H=\bigcap_{i=0}^{\infty}{ }^{i} H=1$, by Baer [2, p. 306] the intersection of all normal subgroups $X$ of $H$ with finite nilpotent factorgroup $H / X$ is 1 . By (c) ${ }^{n} H \leqq X$ for all these $X$; therefore ${ }^{n} H=1$, i.e. $H$ is nilpotent. By (a) and Baer [2, Satz B, p. 299] $H$ is noetherian, i.e. $H$ is noetherian and nilpotent, a contradiction. Q.E.D.

\section{BIBLIOGRAPHY}

Reinhold Baer

1. Nilgruppen, Math. Z. 62 (1955), 402-437.

2. Das Hyperzentrum einer Gruppe III, Math Z. 59 (1953), 299-338.

3. Gruppen mit Minimalbedingung, Math. Ann. 150 (1963), 1-44. Philip Hall

1. The Frattini subgroup of finitely generated groups, Proc. London Math. Soc. (3) 11 (1961), 327-352.

S. S. Kemhadze

1. On the definition of nilpotent groups, Amer. Math. Soc. Transl. (2) 46 (1965), 162-164.

M. F. Newman and J. Wiegold

1. Groups with many nilpotent subgroups, Arch. Math. 15 (1964), 241-250.

UNIVERSITY OF MIAMI 